\title{
Vigilancia de la resistencia de Mycobacterium tuberculosis a las drogas antituberculosas en Cuba, 1995-1998
}

Ernesto Montoro, Miguel Echemendía, Dihadenys Lemus, Antonio Marrero, María J. Llanes, José A. Valdivia

Laboratorio Nacional de Referencia e Investigaciones en Tuberculosis y Micobacterias, Centro Colaborador OPS/OMS, Instituto de Medicina Tropical Pedro Kourí, La Habana, Cuba.

La vigilancia de la resistencia a fármacos a través del cultivo y de las pruebas de susceptibilidad in vitro permite conocer la magnitud regional y mundial de la resistencia en tuberculosis. En el presente trabajo se determinó la prevalencia de la resistencia a fármacos antituberculosos en Cuba, durante el periodo 1995-1998 en casos nuevos y en aquéllos que han recibido tratamiento previo. Los resultados incluidos en este estudio forman parte de los dos proyectos mundiales organizados por la OMS/UICTER. La resistencia a los medicamentos se evaluó usando el método de las proporciones en 1.379 cepas de Mycobacterium tuberculosis a los fármacos de primera línea (isoniacida, rifampicina, estreptomicina y etambutol). La resistencia en casos nuevos fue del $8,3 \%$ y $6,5 \%$ y la resistencia múltiple a fármacos (multidrug-resistance, MDR) fue del 0,7\% y $0 \%$ en el primer y segundo estudio, respectivamente. Estos resultados permitieron demostrar la escasa circulación de cepas MDR en Cuba; se reconoció a nivel mundial el buen funcionamiento del Programa Nacional de Control y el éxito de la aplicación en nuestro país de la estrategia del tratamiento estrictamente supervisado desde 1971.

Palabras clave: resistencia, Mycobacterium tuberculosis, multidrug-resistance.

Surveillance of resistance of Mycobacterium tuberculosis to anti-TB drugs in Cuba, 19951998

Drug-resistance surveillance by means of culture and drug susceptibility test (DST) allows the estimation of the regional and global magnitude of multidrug-resistance (MDR). Two reports on anti-tuberculosis drug resistance by the WHO/IUATLD showed that drug-resistance TB varied widely across regions.

This work determined the prevalence of drug resistance among new cases and among previously treated cases of tuberculosis in Cuba during the period 1995-1998. Drug resistance was determined using the proportion method in 1379 Mycobacterium tuberculosis strains to first line antituberculosis drugs (isoniazid, streptomycin, ethambutol and rifampicin). The resistance in new cases was $8.3 \%$ and $6.5 \%$; MDR was $0.7 \%$ and $0 \%$ in the first and second study respectively. The contributed data through Cuba demonstrated that our country is relatively free of MDR strains, recognizing it to world level the good tuberculosis-control programs and the success of the application in our country of the directly-observed-treatment strategy from the year 1971 .

Keys words: resistance, Mycobacterium tuberculosis, Multidrug-resistant

Una tercera parte de la población mundial está infectada por Mycobacterium tuberculosis (1.722 millones). Se estima que para el 2020 alrededor

\section{Correspondencia:}

Ernesto Montoro, Autopista Novia del Mediodia Km 6 1⁄2,

La Lisa, La Habana, Cuba.

Teléfono: (537) 202 0448; fax: (537) 2040651. emontoro@ipk.sld.cu

Recibido: 26/06/03; aceptado: 26/02/04 de 1.000 millones de personas podrían contraer la infección, 200 millones desarrollar la enfermedad y unos 35 millones morir a causa de la tuberculosis. En el 2000, se declararon 8,4 millones de casos nuevos de tuberculosis en el mundo; si este incremento continúa, se producirán 10,2 millones de casos nuevos hacia el 2005. Cada año mueren 2 millones de personas por causa de la tuberculosis. Estas defunciones representan en 
los países en desarrollo el $25 \%$ del total de muertes evitables en adultos. Noventa y cinco por ciento de los casos de tuberculosis y $98 \%$ de las defunciones causadas por esta enfermedad, se registran en estos países $(1,2)$.

Entre las principales causas que han favorecido el aumento de la tuberculosis a nivel mundial, se encuentra la aparición y circulación de cepas resistentes a múltiples fármacos (multidrugresistance, MDR). La OMS ha estimado que existen en el mundo 50 millones de personas infectadas por bacilos tuberculosos con MDR. Esta situación, de consecuencias impredecibles, puede ser en el futuro una epidemia de tuberculosis potencialmente incurable en la mayoría del planeta, por lo que se ha declarado un estado de emergencia global que debe tenerse en cuenta para abordar medidas urgentes en los diferentes aspectos de la lucha antituberculosa que enfrenta cada país $(3,4)$.

En la región de las Américas, los resultados obtenidos durante la ejecución de los dos proyectos mundiales han permitido conocer que la carga de MDR tiene una limitada importancia (inferior al 3\%), con excepción de Argentina y República Dominicana. Los países con una larga historia de buen desempeño del Programa Nacional de Control (PNC), como Cuba, Chile y Uruguay están relativamente libres de MDR (5).

El siguiente trabajo consiste en la presentación de los resultados de las pruebas de resistencia de Mycobacterium tuberculosis realizadas en el Laboratorio Nacional de Referencia e Investigaciones en Tuberculosis y Mycobacteria (LNR-TB) del Instituto Pedro Kourí (IPK), frente a los fármacos antituberculosos de primera línea durante el período 1995-1998. Estos resultados forman parte de los dos proyectos mundiales (1997 y 2000) organizados por la OMS/UICTER, del cual formamos parte como grupo de trabajo mundial (6-9).

\section{Material y métodos}

Previo a la selección de las cepas, se realizó un método de muestreo de acuerdo con las directrices de la OMS/UICTER sobre vigilancia de la resistencia a fármacos; la selección de las muestras fue por conglomerado en proporción a la población (10).

Se estudiaron 1.379 cepas procedentes de todo el país, lo cual representó del $40 \%$ al $50 \%$ del total de pacientes con baciloscopía positiva de acuerdo con la notificación realizada en cada año de estudio. El método utilizado para la determinación de la resistencia frente a isoniacida (INH), rifampicina (RMP), etambutol (EMB) y estreptomicina (SM) fue el proporcional de Canetti, en su variante económica tipo b mediante la técnica indirecta (11).

Los resultados de resistencia se expresaron en porcentaje para cada medicamento y se dividieron en dos grupos según los criterios actuales de clasificación: resistencia en casos nuevos y resistencia en casos previamente tratados. Se realizó el cálculo del intervalo de confianza del $95 \%$.

\section{Resultados}

De las 786 cepas estudiadas correspondientes al periodo 1995-96, 763 pertenecían a pacientes clasificados como casos nuevos; de ellas, 700 $(91,7 \%)$ resultaron sensibles a los cuatro medicamentos y 63 cepas $(8,3 \%)$ presentaron algún tipo de resistencia. Cuando analizamos el comportamiento de las cepas resistentes frente a cada medicamento, vemos que 55 cepas $(7,2 \%)$ presentaron resistencia a un fármaco; la SM obtuvo la mayor cifra $(6 \%)$, seguida de la INH con $1 \%$; sólo 1 cepa $(0,1 \%)$ fue resistente a RMP (cuadro 1).

Con respecto a la presencia de MDR, se reportaron 5 cepas $(0,7 \%)$. La asociación más frecuente observada fue INH más RMP más SM en 4 cepas $(0,5 \%)$. El resto de los patrones de resistencia obtenidos (INH más SM y RMP más SM) no mostró importancia, pues sólo se halló en 3 cepas (cuadro 1).

Con respecto a la resistencia en pacientes previamente tratados, 23 cepas fueron estudiadas, de las cuales, sólo $2(8,7 \%)$ fueron sensibles y $21(91,3 \%)$ resistentes. La resistencia a un fármaco estuvo presente en 15 cepas $(65,2 \%)$; la más frecuente fue SM con 13 cepas $(56,5 \%)$, seguido de la INH con sólo 2 cepas (8,7\%); ninguna cepa 
Cuadro 1. Prevalencia de resistencia a medicamentos antituberculosos. Cuba, 1995-96.

\begin{tabular}{|c|c|c|c|c|c|c|}
\hline & \multicolumn{2}{|c|}{ Casos nuevos } & \multicolumn{2}{|c|}{$\begin{array}{c}\text { Casos previamente } \\
\text { tratados }\end{array}$} & \multicolumn{2}{|c|}{ Combinada } \\
\hline & $\mathbf{n}$ & $\%$ & $\mathbf{n}$ & $\%$ & $\mathbf{n}$ & $\%$ \\
\hline Total de cepas estudiadas & 763 & 100 & 23 & 100 & 786 & 100 \\
\hline Sensibles & 700 & 91,7 & 2 & 8,7 & 702 & 89,3 \\
\hline Cualquier resistencia & 63 & 8,3 & 21 & $91, .3$ & 84 & 10,7 \\
\hline $\begin{array}{l}\text { Resistencia a } 1 \text { medicamento } \\
\text { INH } \\
\text { RMP } \\
\text { EMB } \\
\text { SM }\end{array}$ & $\begin{array}{c}55 \\
8 \\
1 \\
0 \\
46\end{array}$ & $\begin{array}{l}7,2 \\
1,0 \\
0,1 \\
0,0 \\
6,0\end{array}$ & $\begin{array}{c}15 \\
2 \\
0 \\
0 \\
13\end{array}$ & $\begin{array}{c}65,2 \\
8,7 \\
0,0 \\
0,0 \\
56, .5\end{array}$ & $\begin{array}{c}70 \\
10 \\
1 \\
0 \\
59\end{array}$ & $\begin{array}{l}8,9 \\
1,3 \\
0,1 \\
0,0 \\
7,5\end{array}$ \\
\hline $\begin{array}{l}\text { MDR } \\
\text { INH + RMP } \\
I N H+R M P+S M \\
I N H+R M P+E M B+S M\end{array}$ & $\begin{array}{l}5 \\
1 \\
4 \\
0\end{array}$ & $\begin{array}{l}\mathbf{0 , 7} \\
0,1 \\
0,5 \\
0,0\end{array}$ & $\begin{array}{l}3 \\
0 \\
3 \\
0\end{array}$ & $\begin{array}{c}13,0 \\
0,0 \\
13,0 \\
0,0\end{array}$ & $\begin{array}{l}8 \\
1 \\
7 \\
0\end{array}$ & $\begin{array}{l}1,0 \\
0,1 \\
0,9 \\
0,0\end{array}$ \\
\hline $\begin{array}{l}\text { Otros patrones } \\
\text { INH + SM } \\
\mathrm{RMP}+\mathrm{SM}\end{array}$ & $\begin{array}{l}3 \\
2 \\
1\end{array}$ & $\begin{array}{l}\mathbf{0 , 4} \\
0,3 \\
0,1\end{array}$ & $\begin{array}{l}3 \\
2 \\
1\end{array}$ & $\begin{array}{c}13,0 \\
8,7 \\
4,3\end{array}$ & $\begin{array}{l}6 \\
4 \\
2\end{array}$ & $\begin{array}{l}\mathbf{0 , 8} \\
0,5 \\
0,3\end{array}$ \\
\hline
\end{tabular}

resultó resistente a RMP. Se observaron solamente 3 cepas $(13 \%)$ con MDR; el resto de los patrones se comportó de igual forma que en la resistencia en casos nuevos (cuadro 1).

En el segundo período de estudio correspondiente a 1997-1998, 525 cepas pertenecían a casos nuevos, de las cuales, 491 (93,5\%) eran sensibles a los cuatro medicamentos y $34(6,5 \%)$ resistentes. El 5,7\% de las cepas presentó resistencia a un solo fármaco; la SM $(4,9 \%)$ y la INH $(0,8 \%)$ fueron las únicas que mostraron estos patrones (cuadro 2). No se reportaron cepas MDR. Sólo se obtuvieron 4 cepas con otra combinación de patrones de resistencia (INH más SM y EMB más SM) (cuadro 2).

Con respecto a la resistencia en casos previamente tatados, 68 cepas se incluyeron en esta categoría; de ellas, 46 eran sensibles $(67,6 \%)$ y 22 resistentes $(32,4 \%)$. La resistencia a un medicamento fue de $17,6 \%$ (11,7\% a SM y $5,9 \%$ a INH). La MDR alcanzó un valor del 11,7\% (8 cepas) y el resto de los patrones representó solo el $3 \%$ (cuadro 2 ).

\section{Discusión}

Hasta hace poco, la magnitud del problema de la resistencia a los fármacos antituberculosos no estaba bien definida. Una revisión de la literatura y de reportes no publicados en las últimas décadas sugiere altos niveles de resistencia en algunas áreas. Sin embargo, mucho de estos estudios se hicieron con muestras no representativas o en pacientes de quienes no se conocía el antecedente de tratamiento previo o si correspondía a casos nuevos. La existencia de estas limitaciones alertó sobre la necesidad de adelantar estudios mundiales para conocer y poder comparar los resultados obtenidos entre los diferentes países participantes (7).

Como antecedente de estudios de resistencia realizados en Cuba, se cuenta con el reporte de Valdivia y Suárez Méndez en 1968, en el cual se estudiaron 1.022 cepas de pacientes procedentes de La Habana. Se notificó una resistencia en casos nuevos del $8,6 \%$ frente a la INH, SM y ácido paraaminosalicílico (PAS); la INH fue el medicamento que tuvo la mayor resistencia $(3,8 \%)$. La resistencia en casos previamente tratados fue de $90,8 \%$ : INH con $7,8 \%$ y $48,2 \%$ de las cepas resultó resistente a los tres fármacos evaluados $(\mathrm{INH}$, SM y PAS) (12).

Desde 1982, el LNR-TB del IPK comenzó un estudio longitudinal de la resistencia, paralelo al PNC con el fin de conocer sus magnitudes y variaciones, aspectos relacionados con el tratamiento masivo, su eficacia y control. Se 
Cuadro 2. Prevalencia de resistencia a medicamentos antituberculosos, Cuba, 1997-1998.

\begin{tabular}{lrrrrrr}
\hline & \multicolumn{2}{c}{ Casos nuevos } & \multicolumn{2}{c}{$\begin{array}{c}\text { Casos previamente } \\
\text { tratados }\end{array}$} & \multicolumn{2}{c}{ Combinada } \\
& $\mathbf{n}$ & $\%$ & $\mathbf{n}$ & $\%$ & $\mathbf{n}$ & $\%$ \\
\hline Total de cepas estudiadas & $\mathbf{5 2 5}$ & $\mathbf{1 0 0}$ & $\mathbf{6 8}$ & $\mathbf{1 0 0}$ & $\mathbf{5 9 3}$ & $\mathbf{1 0 0}$ \\
Sensibles & 491 & 93,5 & 46 & 67,6 & 537 & 90,6 \\
Cualquier resistencia & 34 & 6,5 & 22 & 32,4 & 56 & 9,4 \\
Resistencia a 1 medicamento & $\mathbf{3 0}$ & $\mathbf{5 , 7}$ & $\mathbf{1 2}$ & $\mathbf{1 7 , 6}$ & $\mathbf{4 2}$ & $\mathbf{7 , 1}$ \\
INH & 4 & 0,8 & 4 & 5,9 & 8 & 1,3 \\
RMP & 0 & 0,0 & 0 & 0,0 & 0 & 0,0 \\
EMB & 0 & 0,0 & 0 & 0,0 & 0 & 0,0 \\
SM & 26 & 4,9 & 8 & 11,7 & 34 & 5,7 \\
MDR & $\mathbf{0}$ & $\mathbf{0 , 0}$ & $\mathbf{8}$ & $\mathbf{1 1 , 7}$ & $\mathbf{8}$ & $\mathbf{1 , 3}$ \\
INH + RMP & 0 & 0,0 & 2 & 2,9 & 2 & 0,3 \\
INH + RMP + EMB & 0 & 0,0 & 2 & 2,9 & 2 & 0,3 \\
INH + RMP + SM & 0 & 0,0 & 4 & 5,9 & 4 & 0,7 \\
INH + RMP + EMB + SM & 0 & 0,0 & 0 & 0,0 & 0 & 0,0 \\
Otros patrones & $\mathbf{4}$ & $\mathbf{0 , 8}$ & $\mathbf{2}$ & $\mathbf{3 , 0}$ & $\mathbf{6}$ & $\mathbf{1 , 0}$ \\
INH + SM & 3 & 0,6 & 1 & 1,5 & 4 & 0,7 \\
RMP + SM & 0 & 0,0 & 1 & 1,5 & 1 & 0,2 \\
EMB + SM & 1 & 0,2 & 0 & 0,0 & 1 & 0,2 \\
\hline
\end{tabular}

registró una resistencia en casos nuevos del $6 \%$ en 430 cepas estudiadas (1978-1980) frente a los 4 medicamentos [INH, SM, PAS y tiocetazona $\left.\left(\mathrm{tb}_{1}\right)\right]$. El 3,5\% fue resistente a INH y el 5,3\% a la $\mathrm{SM}$. No se encontró resistencia al PAS ni al Tb, y no estuvo presente en dos o más fármacos. La resistencia en casos previamente tratados fue del $5,3 \%$; la resistencia de $4,2 \%$ para INH; de $4,4 \%$ a la SM; de $1,8 \%$ al PAS, y de $0,7 \%$ al $\mathrm{Tb}_{1}(13)$.

No es posible establecer una comparación con relación a los valores de resistencia en casos previamente tratados, entre los trabajos relacionados anteriormente y los actuales. Estos últimos valores no corresponden a un aumento de este tipo de resistencia, sino que son producto de la adopción de los nuevos criterios recomendados en las guías de la OMS/UICTER para la vigilancia de la resistencia (10). En nuestro país existe un número menor de pacientes que son sometidos a retratamientos y un número mucho menor de ellos resulta resistente.

En Cuba, de acuerdo con las etapas por las cuales ha transitado el PNC desde su creación en 1963, se han adoptado diferentes estrategias de tratamiento. En la primera etapa (1964-1971) se obtuvo una curación del $77 \%$ en casos nuevos y alrededor de $50 \%$ en casos crónicos. Durante la segunda etapa (1971-1987), se alcanzaron valores de curación entre $85 \%$ a $92 \%$ y en la tercera etapa (1987, hasta el presente), se han obtenido tasas de curación del 95\%. Desde 1971, se puso en práctica en nuestro país una estrategia integrada de diagnóstico bacteriológico y tratamiento estrictamente supervisado y empezaron a notificarse altos valores de curación y, por consiguiente, se obtienen bajas cifras de resistencia (14).

En estos dos proyectos organizados por la OMS/ UICTER estuvieron representadas todas las regiones de la OMS (35 países en 1997 y 72 en 2000). Este estudio permitió conocer el estado actual de las resistencias en el mundo y, sobre todo, de la MDR. Se pudo llegar a la conclusión de que este fenómeno está presente en todo el mundo, aunque existen variaciones de unas zonas a otras, siempre en estrecha relación con buenos o malos PNC aplicados en el pasado. Así, existen las denominadas 'zonas calientes', con una elevada MDR en casos nuevos (situación más grave), encabezadas por las repúblicas bálticas ex soviéticas (Letonia, 14,4 \%; Estonia, 10,2\%), República Dominicana, 6,6\%, y otros países en los que se han administrado fármacos indiscriminadamente con muy escaso control en 
las últimas décadas (Costa de Marfil, 5,3\%; Argentina, 4,6\%; Rusia, 4\%; Tailandia, 3,8\%; Rumania, 2,8\%; Perú, 2,5\%) (6-9,15-17).

En el polo opuesto están los países que apenas tienen MDR en casos nuevos. Son aquéllos en los que se han seguido buenos PNC; entre ellos se encuentra Cuba con 0\% de MDR en 1998 y otros países en los que se reconocen bajas cifras de resistencia (Escocia, 0,3\%; Francia, 0,5\%, y República Checa, 1\%) (6-9,15-17).

En conclusión, los datos aportados por Cuba permitieron realizar el perfeccionamiento en la vigilancia y control de la resistencia en el LNRTB; la adopción de los criterios internacionales y la obtención de resultados altamente confiables y comparables con otros países del mundo; además, se logró un impacto favorable en el PNC y se demostró que nuestro país se encuentra relativamente libre de cepas MDR, en contraste con la situación alarmante que presentan otros países mencionados anteriormente.

\section{Referencias}

1. World Health Organization. Factsheet 2000;104

2. World Health Organization. Global DOTS expansion plan. Progress in $\mathrm{Tb}$ control in high-burden countries, WHO/CDS/STB/2001.11. Geneva: World Health Organization; 2001. p.35-9.

3. World Health Organization. Global tuberculosis control. WHO report 2000. WHO/CDS/TB/2000.275. Geneva: World Health Organization; 2000. p.87-102.

4. World Health Organization. Stop TB annual report 2000. Forging new partnerships to stop TB. WHO/CDS/ STB/2001.12. Geneva: World Health Organization; 2001. p.3-11.

5. Organización Panamericana de la Salud. Magnitud de la resistencia a los fármacos anti-TB en la región de las Américas. Tuberculosis 2000;3:1-2.

6. World Health Organization. Anti-tuberculosis drug resistance in the world: the WHO/IUATLD global project on anti-tuberculosis drug resistance surveillance 1994-
1997. WHO/TB/97.227. Geneva: World Health Organization; 1997.

7. Pablos-Méndez A, Raviglione MC, Laszlo A, Binkin N, Rieder HL, Bustreo F, et al. Global surveillance for anti-tuberculosis drug resistance 1994-1997. N Engl J Med 1998;338:1641-9.

8. World Health Organization. Anti-tuberculosis drug resistance in the world: report No. 2. Prevalence and trends. The WHO/IUATLD global project on anti-tuberculosis drug resistance surveillance. WHO/TB/ 2000.278. Geneva: World Health Organization; 2000.

9. Espinal M, Laszlo A, Simonsen L, Bonlahbal F, Kim SJ, Reniero A, et al. Global trends in resistance to anti-tuberculosis drugs. N Engl J Med 2001;344:1294303.

10. Bustreo F, Pablos-Méndez A, Raviglione M, Murria J, Trébucq A, Rider H. Directrices para la vigilancia de la farmacorresistencia en la tuberculosis. WHO/TB/ 96.216. Ginebra: OMS/UICTER; 1997. p.5-25.

11. Canetti G, Fox W, Khomenko Al. Advances in techniques of testing mycobacterial drug sensitivity and the use of sensitivity tests in tuberculosis control programs. Bull World Health Organ 1969;41:21-43.

12. Valdivia JA, Suárez R. Incidencia y prevalencia de cepas drogorresistentes en el hospital antituberculoso Julio Trigo de La Habana. Bol Hig Epid 1968;6:247-51.

13. Valdivia JA, Jiménez C, Rodríguez R, Mederos L, Echemendía $\mathbf{M}$, Valdés $\mathbf{L}$ y cols. Estudio cooperativo de la resistencia a las drogas antibacilares en cepas de $M$. tuberculosis aisladas en pacientes tuberculosos. Rev Cubana Med Trop 1982;34:119-25.

14. Marrero A, Caminero JA, Rodríguez R, Billo NE. Towards elimination of tuberculosis in a low income country: the experience of Cuba, 1962-1997. Thorax 2000;55:39-45.

15 Caminero JA. Origen, presente y futuro de las resistencias en tuberculosis. Arch Bronconeumol 2001;37:35-42.

16. Montoro E, Echemendía M, Lemus D, Valdivia JA. Estudio de la resistencia del Mycobacterium tuberculosis en Cuba 1997-98. Avances Biotecnología Moderna 1999;5:D10.

17. Montoro E. Resistencia de Mycobacterium tuberculosis en Cuba y las Américas. Rev Latinoam Microbiol 2000; 42:117.

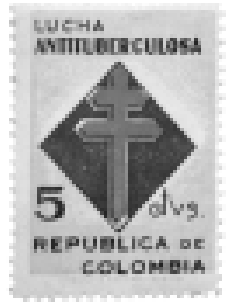

